\title{
Google lo sa
}

\section{Google knows it}

Giovanni Lodi

Dipartimento di Scienze Biomediche, Chirurgiche e Odontoiatriche

Università degli Studi di Milano

via Beldiletto 1/3 Milano 20142

Italia

giovanni.lodi@unimi.it

"A quanti studi sperimentali pensi di aver partecipato nell'ultimo anno?"

Comincia così, con una domanda diretta al lettore, il libro The power of experiments di Michael Luca e Max $\mathrm{H}$ Bazerman, docenti di economia ad Harward. La risposta che ci viene data nelle righe successive è piuttosto sorprendente: "A meno che tu non sia vissuto in un bunker senza accesso a internet, hai partecipato a molti trial". Perché leggendo, scopriamo che chiunque di noi abbia una pagina di Facebook, compri su Amazon, venda su eBay o sia abbonato a Netflix, è stato probabilmente soggetto inconsapevole di più di uno studio controllato.

Le mega aziende hi-tech hanno compreso la forza del trial randomizzato, che la medicina usa (o dovrebbe usare) per stabilire se un trattamento funziona, e hanno iniziato ad utilizzarlo per influenzare le nostre scelte, testando le caratteristiche di un sito capaci di catturare la nostra attenzione o il modo migliore per informarci sul prezzo di ciò che stiamo comprando. Non ci sono comitati etici, i partecipanti sono decine di migliaia, i dati abbondantissimi e di conseguenza i risultati molto affidabili. Considerando che spesso ciò si traduce in un incremento dei profitti, non c'è da stupirsi che Google abbia condotti più di 10.000 trial nel 2018. E non ci sono solo le aziende del web: il fisco inglese, grazie a uno studio sul testo di una lettera indirizzata agli evasori, ha aumentato significativamente le proprie entrate.

Condurre trial è diventato anche POP. Proprio mentre stavo preparando questo editoriale (sarà un caso?), Netflix mi ha proposto una serie dal titolo 100 Humans in cui tre ricercatori rispondono a domande sui comportamenti umani servendosi di cento volontari. Se avete un po' di tempo da perdere guardatela, è piuttosto divertente, sebbene il disegno degli studi non sia sempre rigoroso.

Efficace, ubiquitario, utile, redditizio, POP, è il trial randomizzato. Eppure, proprio tra chi si occupa di salute, sono ancora in molti a preferire esperienza personale, intuito e consuetudine.

Buona lettura, 